\title{
PARASITE CO-STRUCTURE: BROAD AND LOCAL SCALE APPROACHES
}

\author{
CRISCIONE C.D.****
}

\section{Summary:}

A co-structure study is a comparison of demographic and/or genetic structure between two or more species. Such a comparative analysis among a parasite and its host(s) or among multiple parasite species is useful to elucidate factors that shape genetic variation within and among parasite populations. I provide a brief review of how co-structure studies in parasite systems can be used to address ecological, evolutionary, and epidemiological questions. Subjects that can be addressed with parasite costructure studies range from broad-scale analyses that compare phylogeographical patterns to local scale analyses that examine among host transmission within a host population.

KEY WORDS : co-structure, comparative phylogeography, molecular epidemiology, parasite, population genetics.

$\mathrm{H}$ ere, I provide a mini-review of parasite studies that use co-structure analyses to examine genetic variation within and among parasite populations. I broadly define a co-structure study as a comparison of population demographic and/or genetic structures between two or more species with the aim of elucidating factors that determine the population demographic and/or genetic structure in one or more of the species under comparison. Thus, comparisons between parasites and their hosts and comparisons of two or more parasite species are considered co-structure analyses. My aim is to highlight how comparative analyses can be used to address ecological, evolutionary, and applied questions in parasite systems. The focus is largely on factors that affect parasite gene flow, genetic drift, mating systems, or among host transmission. I first discuss questions at a broad scale (i.e., among geographic regions) and then on a local scale (i.e., within a host population in a given geographic location).

\footnotetext{
* Department of Genetics, Southwest Foundation for Biomedical Research. P.O. Box 760549, San Antonio, Texas, 78245-0549, USA. ** Department of Biology, 3258 TAMU, Texas A\&M University, College Station, TX 77843, USA (as of August 2008).

Tel.: 2102589722 - Fax: 2106703344.

Email: ccriscione@mail.bio.tamu.edu
}

\section{BROAD SCALE ANALYSES}

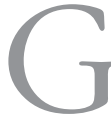
ene flow and population history are major fac-tors that affect genetic structure among populations and potentially, local adaptation within populations. Thus, comparisons of genetic structure on a broad scale are largely designed to test factors that control dispersal, reconstruct historical events (e.g., vicariance events, post-glacial colonization routes), or compare relative rates of gene flow between a host and its parasite. In this section, I highlight the above subjects, plus illustrate some additional topics that can be covered with co-structure studies at the broad scale. Comparing the genetic structure among populations of multiple species that differ in key traits is useful to elucidate natural history characteristics that affect dispersal among populations. Data from several parasiteparasite studies indicate that host movement is a key determinant of parasite gene flow (Blouin et al., 1995; McCoy et al., 2003a; Criscione \& Blouin, 2004). For instance, trematode species that cycle exclusively in freshwater hosts are much more genetically subdivided among rivers than another fluke from the same locations, but whose life cycle includes highly mobile terrestrial hosts (Criscione \& Blouin, 2004). Johnson et al. (2002) found differences in genetic structure among species of dove lice. In part, these patterns may be due to the ability of some louse species to hitch-hike on hippoboscid flies. Whiteman et al. (2007) showed how differences in natural history traits among parasites of the same host species could lead to testable predictions about the factors that influence among population genetic structure in parasites. In a parasite-multihost comparison, Prugnolle et al. (2005) found that parasite migration was largely influenced by the most vagile host in the parasite's life cycle.

Phylogeographical comparisons among multiple species can identify historically and evolutionarily independent geographic regions. Congruent patterns among species suggest shared biogeographical factors that have shaped intraspecific population histories (Bermingham \& Moritz, 1998; Avise, 2000). Several host-parasite comparisons have used a phylogeographical approach 
to make inferences about parasite population history (Criscione et al., 2005). For example, mitochondrial (mtDNA) data from the nematode Heligmosomoides polygyrus show levels of genetic diversity that support some regions in Europe as glacial refuges and show patterns of postglacial recolonization that parallel those of its wood mouse host (Nieberding et al., 2004; Nieberding et al., 2005). However, the parasite did show strong genetic subdivision among some locations that the host did not (Nieberding et al., 2004). Largely congruent phylogeographical patterns coupled with some parasite specific patterns have also been reported in other host-parasite systems (e.g., Wickstrom et al., 2003; Meinila et al., 2004; Criscione \& Blouin, 2007). Several inferences about parasite ecology and evolution, some with practical applications, can be drawn from congruent host-parasite phylogeographical patterns. 1) Patterns of congruence show that parasites can be affected by the same historical processes that affect their hosts. 2) When a host or geographic region is of conservation concern, parasite data can be used to help support the boundaries of historically unique regions or managed host populations (Criscione \& Blouin, 2007). 3) Comparative host-parasite studies are useful to predict parasite dispersal potential and scale of gene flow over a broad geographical range (Criscione \& Blouin, 2007). 4) Congruent patterns can be used to calibrate a molecular clock for parasites (Meinila et al., 2004; Nieberding et al., 2004). This is particularly useful as many parasitic taxa have no or few fossil representatives.

Incongruent patterns between host and parasite also shed light on parasite ecology and evolution. Whether a host and parasite show congruence will depend on which host in a parasite's life cycle is compared and on the nature of the transmission dynamics between the host and parasite (Nierberding \& Olivieri, 2007). For example, malaria parasites of Caribbean lizards showed complex patterns of genetic fragmentation that were inconsistent with those of their lizard hosts. Thus, vector hosts likely provided an effective means of dispersal among islands for the malaria parasites (Perkins, 2001). It has recently been advocated that when patterns of genetic structure are observed in parasites, but not in their hosts, inferences about cryptic host population history or migration patterns can be drawn (Whiteman \& Parker, 2005; Wirth et al., 2005; Biek et al., 2006; Nieberding \& Olivieri, 2007). However, such data should only be used as a means to generate hypotheses about host history rather than drawing definitive conclusions from the history of a single parasite. This is because many processes can make the parasite have a different pattern of genetic structure than its host. For example, host gene flow does not necessarily equate to parasite gene flow because not all hosts are infected, parasites may be locally adapted in ways that hosts are not, and effective sizes of host and parasite may be very different. Inferences of host history from a single parasite's phylogeography suffer from all the caveats of using a single gene tree to infer a species tree (Nichols, 2001). For example, one can be mislead by processes (e.g., selection, introgression) that affect the mtDNA differently from the nuclear genome (see Ballard \& Whitlock, 2004). Thus, in order to test host history with parasite data, multiple parasite species from a single host species would need to be compared.

Theoretical models predict that local adaptation by parasites to their host is facilitated by higher parasite gene flow because novel alleles that may counteract a host's evolutionary response are imported into the population (Gandon \& Michalakis, 2002). A recent meta-analysis by Greischar et al. (2007) provides empirical support for this prediction. Thus, host-parasite co-structure studies that determine the relative migration rates between a parasite and its host can help predict the potential for local adaptation. Although additional data are needed to demonstrate local host adaptation (e.g., experimental infections), knowledge of relative gene flow rates is now seen as an essential component in testing coevolutionary hypotheses (Gomulkiewicz et al., 2007). See Greischar et al. (2007) for a review of studies comparing host-parasite gene flow in relation to local adaptation.

Additional topics that use host-parasite co-structure analyses are illustrated with the following studies. McCoy et al. (2001) found evidence for sympatric host races by comparing the genetic structure of the assumed generalist tick parasite Ixodes uriae among two seabird species. There was higher genetic subdivision between tick populations from the different host species in sympatry than between allopatric tick populations of the same host species. Miura et al. (2006) examined colonization dynamics by comparing the genetic diversity of an introduced snail and its trematode parasites. Molecular data indicated a founder effect in the snail and one of its flukes. However, another trematode species had similar levels of diversity to its native range, which suggests that this fluke can continually disperse from its native range in Japan to North America via bird hosts. Criscione et al. (2006) show that parasite genetic markers can be more accurate than host genetic markers in assigning host individuals back to their population of origin. It is important to note that this unique application is not inferring host historical events, but rather identifies the discrete genetic parasite population from which a single host became infected. Genetic assignment of parasites may be useful to identify dispersal patterns or feeding grounds for migratory host species, or in epidemiological studies to indicate foci of transmission for infected hosts.

\section{LOCAL SCALE}

Levels and patterns of genetic diversity within populations are influenced by the effective population size 
$\left(N_{e}\right)$ and mating system (outcrossing, selfing, biparental inbreeding). By limiting conspecific interactions to within hosts, the distribution of parasites among individual hosts can affect both $N_{e}$ and mating dynamics (Criscione \& Blouin, 2005; Criscione et al., 2005). For example, an obligatorily sexually reproducing hermaphroditic parasite that is alone in a host must self to reproduce. Moreover, the distribution of parasite genetic variation within and among hosts can be indicative of the ecology of transmission from host to host (Fig. 1) (Criscione et al., 2005; Criscione \& Blouin, 2006). In this section, I discuss how co-structure studies at a local scale can be used to elucidate factors that affect genetic diversity, mating systems, or transmission dynamics within a parasite component population (all parasites of a species within a host population).

As at the broad scale, local scale comparisons of parasites that differ in some aspect of their natural history can provide insight into determinants of within population genetic structure. Parasite-parasite comparisons would be useful to see if mating systems or levels of

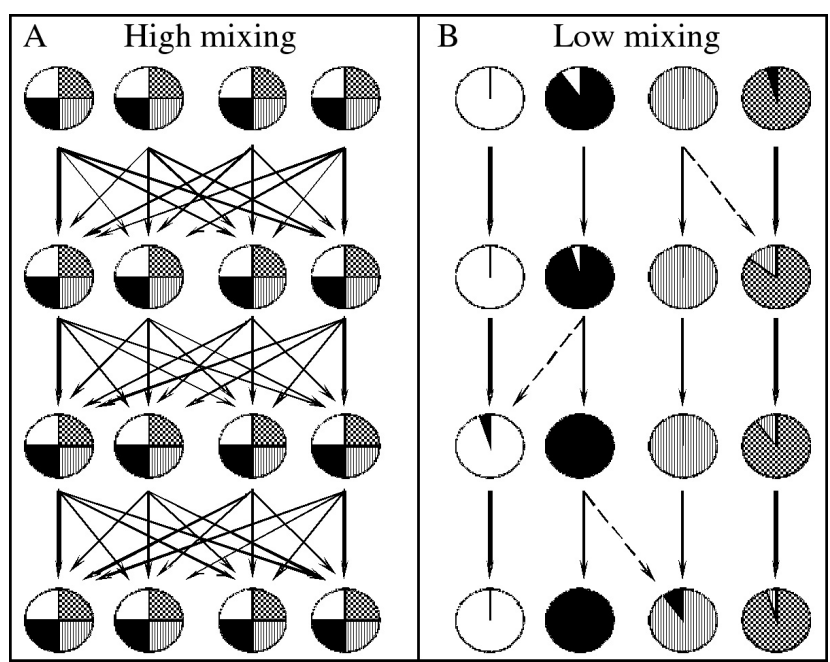

Fig. 1. - Schematic showing how patterns of parasite genetic variation among hosts can be used to infer the transmission process. Circles represent individual definitive hosts. Patterns within circles are different parasite genetic variants. Four generations (rows from top to bottom) of adult parasites and hosts are illustrated. Dashed and solid arrows indicate limited and major paths of recruitment for parasite offspring into definitive hosts, respectively. Parasite offspring may pass through intermediate hosts before reaching definitive hosts. (A) Parasite genetic variation is randomly distributed among hosts with a high amount of mixing among parasite offspring before recruitment into definitive hosts. This pattern indicates that hosts are randomly sampling from a common parasite gene pool. (B) Low mixing of parasite offspring (i.e., clumped transmission) predicts high genetic differentiation among individual hosts. This pattern indicates that hosts are sampling distinct parasite gene pools. These schematics represent the extremes of different transmission processes. In reality, there is likely a continuum among parasite species. Figured was altered from Criscione \& Blouin (2006). See also Criscione et al. (2005), Criscione \& Blouin (2005) and references therein for a more thorough discussion of parasite transmission inferred from genetic markers. diversity vary with regard to mean abundances or prevalence. For example, does the aggregation of parasites among hosts affect the $N_{e}$ (Criscione \&Blouin, 2005)? Would a hermaphroditic species with low mean intensities have a higher selfing rate relative to a species with a high mean intensity (Criscione et al., 2005)? For instance, Anderson et al. (2000) found evidence of inbreeding (low genetic diversity and linkage disequilibrium) in malaria populations of low prevalence. It is commonly found in the plant literature that outcrossing species tend to have higher levels of diversity than selfing species (Charlesworth, 2003). Some nematode parasites appear to have a similar pattern (Blouin, 1998), but more work is needed to generalize these conclusions among parasites. The mode of transmission (e.g., the life cycle pattern, dispersal ability of larvae) may also affect patterns of genetic structure within and among hosts (Fig. 1). For instance, would roundworms (e.g., Ascaris) show more structure (Fig. 1B) because transmission is via passive oral infection, whereas hookworms (e.g., Necator) actively penetrate the host's skin? Also, parasite-parasite comparisons could be used to test the hypothesis that parasites in an aquatic environment and/or those using multiple intermediate hosts will have greater mixing potential into definitive hosts (Fig. 1A) (Criscione \& Blouin, 2006). Determining focal points of transmission, and factors that influence these foci are of major importance to understanding the ecology and evolution of parasites. In the field of epidemiology, these questions are of practical importance as they help design effective parasite control or eradication programs. Highly polymorphic molecular markers (e.g., microsatellites) and powerful assignment and landscape statistical methods (e.g., Francois et al., 2006; Storfer et al., 2007) make it possible to examine if there are distinct genetic clusters of parasites (i.e., focal points of transmission) within a host population (Fig. 1B). A few studies have begun using these approaches (e.g., McCoy et al., 2003b; Criscione \& Blouin, 2006; Chevillon et al., 2007). However, host-parasite co-structure studies at the local scale will be needed to help tease apart whether host and/or environmental variables have a significant role in shaping the local parasite genetic structure. For example, a correlation in host genetic relatedness to parasite genetic relatedness may suggest that there is a genetic predisposition among hosts to be infected with particular parasite genetic variants. Furthermore, fine scale parasite genetic structure may be related to the demographic structure of host populations such as gender, age, social groups, dominance hierarchies, or territoriality. A few studies have begun using the above approach. Ongoing work utilizes these methods to examine the molecular epidemiology of Ascaris lumbricoides in a village in Nepal (Criscione, Williams-Blangero \& Anderson, unpublished data). Nebavi et al. 
(2006) examined several host variables and found that individual patient and gender were the most important factors contributing to genetic variability in an opportunistic fungal pathogen. Similarly, Caillaud et al. (2006) found that parasite genetic diversity was different among male and female hosts. These latter two studies suggest that there may be differences in immunocompetence between male and female hosts, or that the two sexes are differentially sampling the parasite gene pool. Molecular epidemiology studies on microbial and viral pathogens have also utilized host demographic factors and pathogen genetic data to help construct contact networks between infected people (e.g., Choudhury et al., 2006).

\section{FUTURE DIRECTIONS}

I have provided a brief overview of topics that can be addressed with parasite co-structure studies. The review is not exhaustive in terms of cited literature, taxonomic groups of parasites, or questions that can be addressed. However, it is clear that there is more empirical data at the broad scale level, while local scale studies have received less attention. Much knowledge can be gained about the ecology and evolution from local scale analyses. In particular, molecular epidemiological studies are likely to benefit from local scale analyses that aim to determine if there are focal points of transmission and if so, what are the determinants of those foci. Lastly, I have focused on inferences from neutral loci. However, a comparative framework will also be useful to examine factors that influence the patterns of genetic variation at adaptive loci. For example, how might the distribution of potential host species within and among regions affect genetic variation at parasite loci that confer infectivity (i.e., host-specificity genes)?

\section{ACKNOWLEDGEMENTS}

Trtive thank Mike Blouin and Tim Anderson for constructive comments on the manuscript, and Thierry de Meeûs for the invitation to participate in the EMOP symposia.

\section{REFERENCES}

Anderson T.J.C., Haubold B., Williams J.T., Estrada-Franco J.G., Richardson L., Mollinedo R., Bockarie M., Mokili J., Mharakurwa S., French N., Whitworth J., Velez I.D., Brockman A.H., Nosten F., Ferreira M.U. \& Day K.P. Microsatellite markers reveal a spectrum of population structures in the malaria parasite Plasmodium falciparum. Molecular Biology and Evolution, 2000, 17, 1467-1482.

Avise J.C., Phylogeography: The History and Formation of Species. Harvard University Press, Cambridge, Massachusetts, 2000.
Ballard J.W.O. \& Whitlock M.C. The incomplete natural history of mitochondria. Molecular Ecology, 2004, 13, 729-744.

Bermingham E. \& Moritz C. Comparative phylogeography: concepts and applications. Molecular Ecology, 1998, 7, 367369.

Biek R., Drummond A.J. \& Poss M. A virus reveals population structure and recent demographic history of its carnivore host. Science, 2006, 311, 538-541.

Blouin M.S. Mitochondrial DNA diversity in nematodes. Journal of Helminthology, 1998, 72, 285-289.

Blouin M.S., Yowell C.A., Courtney C.H. \& Dame J.B. Host movement and the genetic structure of populations of parasitic nematodes. Genetics, 1995, 141, 1007-1014.

Caillaud D., Prugnolle F., Durand P., Theron A. \& de Meeûs T. Host sex and parasite genetic diversity. Microbes and Infection, 2006, 8, 2477-2483.

CHARLESWORTH D. Effects of inbreeding on the genetic diversity of populations. Philosophical Transactions of the Royal Society (Series B), 2003, 358, 1051-1070.

Chevillon C., Koffi B.B., Barre N., Durand P., Arnathau C. \& DE MEEÛS T. Direct and indirect inferences on parasite mating and gene transmission patterns - Pangamy in the cattle tick Rhipicephalus (Boophilus) microplus. Infection Genetics and Evolution, 2007, 7, 298-304.

Choudhury B., Risley C.L., Ghani A.C., Bishop C.J., Ward H., FENTON K.A., IsOn C.A. \& SPRATT B.G. Identification of individuals with gonorrhoea within sexual networks: a population-based study. Lancet, 2006, 368, 139-146.

Criscione C.D. \& Blouin M.S. Life cycles shape parasite evolution: comparative population genetics of salmon trematodes. Evolution, 2004, 58, 198-202.

Criscione C.D. \& Blouin M.S. Effective sizes of macroparasite populations: a conceptual model. Trends in Parasitology, 2005, 21, 212-217.

Criscione C.D. \& Blouin M.S. Minimal selfing, few clones, and no among-host genetic structure in a hermaphroditic parasite with asexual larval propagation. Evolution, 2006, 60, 553-562.

CRISCIOnE C.D. \& Blouin M.S. Parasite phylogeographical congruence with salmon host evolutionarily significant units: implications for salmon conservation. Molecular Ecology, 2007, 16, 993-1005.

Criscione C.D., Cooper B. \& Blouin M.S. Parasite genotypes identify source populations of migratory fish more accurately than fish genotypes. Ecology, 2006, 87, 823-828.

Criscione C.D., Poulin R. \& Blouin M.S. Molecular ecology of parasites: elucidating ecological and microevolutionary processes. Molecular Ecology, 2005, 14, 2247-2257.

Francois O., ANCElet S. \& Guillot G. Bayesian clustering using hidden Markov random fields in spatial population genetics. Genetics, 2006, 174, 805-816.

GANDON S. \& Michalakis Y. Local adaptation, evolutionary potential and host-parasite coevolution: interactions between migration, mutation, population size and generation time. Journal of Evolutionary Biology, 2002, 15, 451-462.

Gomulkiewicz R., Brown D.M., Dybdahl M.F., Godsoe W., Nuismer S.L., Pepin K.M., Ridenhour B.J., Smith C.I. \& 
Yoder J.B. Dos and don'ts of testing the geographic mosaic theory of coevolution. Heredity, 2007, 98, 249-258.

Greischar M.A. \& Koskella B. A synthesis of experimental work on parasite local adaptation. Ecology Letters, 2007, 10, 418-434.

Johnson K.P., Williams B.L., Drown D.M., Adams R.J. \& Clayton D.H. The population genetics of host specificity: genetic differentiation in dove lice (Insecta: Phthiraptera). Molecular Ecology, 2002, 11, 25-38.

McCoy K.D., Boulinier T., Tirard C. \& Michalakis Y. Host specificity of a generalist parasite: genetic evidence of sympatric host races in the seabird tick Ixodes uriae. Journal of Evolutionary Biology, 2001, 14, 395-405.

McCoy K.D., Boulinier T., Tirard C. \& Michalakis Y. Hostdependent genetic structure of parasite populations: differential dispersal of seabird tick host races. Evolution, 2003a, 57, 288-296.

McCoy K.D., Tirard C. \& Michalakis Y. Spatial genetic structure of the ectoparasite Ixodes uriae within breeding cliffs of its colonial seabird host. Heredity, 2003b, 91, 422-429.

Meinila M., Kuusela J., Zietara M.S. \& Lumme J. Initial steps of speciation by geographic isolation and host switch in salmonid pathogen Gyrodactylus salaris (Monogenea: Gyrodactylidae). International Journal for Parasitology, 2004, 34, 515-526.

Miura O., Torchin M.E., Kuris A.M., Hechinger R.F. \& Chiba S. Introduced cryptic species of parasites exhibit different invasion pathways. Proceedings of the National Academy of Sciences, 2006, 103, 19818-19823.

Nébavi F., Ayala F.J., Renaud F., Bertout S., Eholié S., Moussa K., Maluí M. \& DE Meeûs T. Clonal population structure and genetic diversity of Candida albicans in AIDS patients from Abidjan (Cote d'Ivoire). Proceedings of the National Academy of Sciences, 2006, 103, 3663-3668.

Nichols R. Gene trees and species trees are not the same. Trends in Ecology and Evolution, 2001, 16, 358-364.

Nieberding C., Libois R., Douady C.J., Morand S. \& Michaux J.R. Phylogeography of a nematode (Heligmosomoides polygyrus) in the western Palearctic region: persistence of northern cryptic populations during ice ages? Molecular Eco$\operatorname{logy}, 2005,14,765-779$.

Nieberding C., Morand S., Libois R. \& Michaux J.R. A parasite reveals cryptic phylogeographic history of its host. Proceedings of the Royal Society of London. Series B, 2004, 271, 2559-2568.

Nieberding C.M. \& Olivieri I. Parasites: proxies for host genealogy and ecology? Trends in Ecology and Evolution, 2007, 22, 156-165.

PERKINS S.L. Phylogeography of Caribbean lizard malaria: tracing the history of vector-borne parasites. Journal of Evolutionary Biology, 2001, 14, 34-45.

Prugnolle F., Théron A., Pointier J.P., Jabbour-Zahab R., Jarne P., Durand P. \& DE MeEÛs T. Dispersal in a parasitic worm and its two hosts: consequence for local adaptation. Evolution, 2005, 59, 296-303.

Storfer A., Murphy M.A., Evans J.S., Goldberg C.S., Robinson S., Spear S.F., Dezzani R., Delmelle E., Vierling L. \& WAITS L.P. Putting the "landscape" in landscape genetics. Heredity, 2007, 98, 128-142.
Whiteman N.K. \& PARKer P.G. Using parasites to infer host population history: a new rationale for parasite conservation. Animal Conservation, 2005, 8, 175-181.

Whiteman N.K., Kimball, R.T. \& Parker P.G. Co-phylogeography and comparative population genetics of the threatened Galápagos hawk and three ectoparasite species: ecology shapes population histories within parasite communities. Molecular Ecology, 2007, 16, 4759-4773.

Wickstrom L.M., Haukisalmi V., Varis S., Hantula J., Fedorov V.B. \& HentTonen H. Phylogeography of the circumpoplar Paranoplocephala artica species complex (Cestoda: Anoplocephalidae) parasitizing collard lemmings (Dicrostonyx spp.). Molecular Ecology, 2003, 12, 3359-3371.

Wirth T., Meyer A. \& Achtman M. Deciphering host migrations and origins by means of their microbes. Molecular Ecology, 2005, 14, 3289-3306. 